\title{
Coccidioidomycosis of the Testis and Epididymis
}

\author{
Dane E. Klett, Y. Mark Hong \\ Department of Urology, Creighton University School of Medicine at St. Joseph's Hospital \& Medical Center, Phoenix, USA \\ Email: dklett0@live.com
}

Received February 27, 2013; revised March 28, 2013; accepted April 5, 2013

Copyright (C) 2013 Dane E. Klett, Y. Mark Hong. This is an open access article distributed under the Creative Commons Attribution License, which permits unrestricted use, distribution, and reproduction in any medium, provided the original work is properly cited.

\begin{abstract}
This report describes an unusual presentation of a man with a testicular mass who presented in diabetic ketoacidosis to the emergency department. Initial diagnosis was a suspected testicular malignancy based on history, physical exam, and available laboratory and radiological imaging. Due to the possibility of a testicular malignancy, the patient was taken for immediate orchiectomy. Histology revealed coccidioidomycosis of the left testicle and epididymis. Treatment with the appropriate anti-fungals was initiated. Despite aggressive multi-disciplinary therapy the patient continued to deteriorate and eventually expired as a result of the massive systemic infection. Literature review revealed the presentation of disseminated genitourinary coccidioidomycosis is rare and seldom reported.
\end{abstract}

Keywords: Coccidioidomycosis; Testicular Mass; Epididymitis; Orchitis; Valley Fever

\section{Introduction}

Coccidioidomycosis is an infection caused by dimorphic fungi of the genus Coccidioides. These fungi are endemic to the southwestern United States, northern Mexico, and Central and South America. The majority of cases, 60\%, are asymptomatic, and the remaining 40\% commonly present with symptoms suggestive of a respiratory infection, most of which are mild and self-limited [1]. Symptomatic extrapulmonary presentations, including disseminated disease, are seen in about 1 in 200 people infected, and these infections most often occur in men, pregnant women, immunocompromised patients, nonwhite individuals, and the elderly [2]. Dissemination occurs via the hematogenous route, and the most common sites include skin, bone, joints, meninges, and soft tissue. Based on postmortem studies, the genitourinary tract is a surprisingly common location of dissemination, with the kidneys being most common, but scrotal disease is rare and represents roughly $1.5 \%$ of all disseminated cases [3]. We present a case of primary pulmonary coccidioidomycosis with subsequent dissemination to the testis and epididymis.

\section{Literature Search}

A search of the medical literature was conducted utilizing the National Library of Medicine (MEDLINE and PubMed). Search terms included testicular mass, testicular pain, testicular infection, testis, testicle, epididymis, epididymitis, orchitis, coccidioidomycosis, coccidia, and valley fever. We identified 25 cases involving the epididymis and/or testes since 1950 [4-21]. Of the 25 cases identified, only 3 were noted to have simultaneous pulmonary infection $[5,10,14]$.

\section{Case Report}

A 44-year old homeless white male with a history of insulin dependent diabetes mellitus and hypertension presented with fatigue, elevated blood glucose and a painless left testicular mass increasing in size over the previous month. Over the previous six months, patient had also experienced progressive weakness, shortness of breath, dry cough, and severe weight loss (60 pounds). He denied fever, chills, chest pain, or night sweats and had not sought medical care until this presentation. On physical exam, the patient appeared cachectic and the left testicle was enlarged, lobulated and non-tender without erythema. The phallus, right testicle and other scrotal structures were unremarkable. Urinalysis and urine culture were negative. Chest imaging revealed diffuse nodular infiltrates bilaterally suggesting metastatic disease versus atypical pneumonia. Scrotal ultrasound revealed a heterogeneous, vascular left testicular mass suggestive of testicular tumor with a normal right testicle. After obtaining informed consent, patient underwent left inguinal orchiectomy.

Surgical exploration revealed a left testicular mass that 
was lobular and heterogeneous. A $4 \mathrm{~cm}$ fluid-filled structure adjacent to left testis expressed pus. Palpation of the right testicle revealed no heterogeneous masses. Left inguinal orchiectomy with high ligation of spermatic cord was performed. Histopathology was negative for malignancy but revealed testicular coccidioidomycosis with necrotizing granulomatous inflammation predominantly involving' the epididymis. Fluconazole and amphotericin B lipid complex therapy were initiated along with the concurrent treatment of multiple medical issues including diabetic ketoacidosis and fatigue requiring ICU care.

Immunological studies including $\operatorname{IgM}$ and $\operatorname{IgG}$ antibodies as well as complement fixation studies were positive for Coccidioides. Testicular tumor markers were negative. Multidisciplinary team management was continued for primary pulmonary coccidioidomycosis with dissemination to the genitourinary tract. Patient continued to deteriorate despite multiple antifungals and antibiotics, experiencing severe complications related to disseminated coccidioidomycosis including respiratory failure requiring ventilator support. Several weeks into his hospital course, patient developed multi-organ failure and rapid decline prompting the patient's family to withdraw care and the patient subsequently expired. Postmortem examination was declined.

\section{Discussion}

Coccidioidomycosis is caused by dimorphic fungi of the genus Coccidioides which are endemic to the southwestern United States, northern Mexico, and Central and South America. It has been estimated there are more than 100,000 new cases of Coccidioides in the US annually [22]. Of these cases, less than $1 \%$ result in symptomatic extrapulmonary dissemination [1]. Even rarer is symptomatic dissemination to the testes and/or epididymis. In post-mortem studies on patients with disseminated disease it was noted that roughly $1.5 \%$ of all disseminated cases result in symptomatic scrotal disease [3]. Risk for disseminated disease is increased in males, African Americans, Filipinos, immunocompromised states, diabetes mellitus, advanced age, and/or pregnancy [1].

In general, diagnosis of coccidioidomycosis requires significant clinical suspicion as it is often misdiagnosed as community-acquired bacterial pneumonia [1]. Diagnoses can be established utilizing the overall clinical picture including the probability of exposure, chest x-ray, coccidioidin skin test (positive with any prior exposure), complement fixation studies, and measurement of IgM and IgG antibodies to the coccidioidin antigen. Definitive diagnosis can be established via biopsy and/or fungal culture. Once the diagnosis of coccidioidomycosis is established it is important to explore the possibility of dissemination. Specifically, dissemination to the male reproductive tract may be associated with a history of voiding dysfunction or scrotal swelling, or a urinalysis indicating hematuria, pneumaturia, coccidiouria or concomitant bacilluria [3]. Diagnosis can be definitively established by utilizing histological study of tissue or urine culture [3]. After diagnosis has been established it is important to begin treatment immediately as morbidity and mortality may be high if delayed. The first line treatment for active or disseminated coccidioidomycosis, including the male genitourinary tract, is systemic antifungal therapy with an azole or a lipid formulation of amphotericin B [3]. When infected scrotal contents are involved, as was the case with our patient, surgical resection has been advocated in addition to the systemic anti-fungal treatment.

We present a rare case of symptomatic, primary pulmonary coccidioidomycosis with simultaneous presentation of a testicular mass due to dissemination. The differential diagnosis for this mass includes: primary testicular tumor, abdominal hernia, lymphoma, primary bacterial or fungal infection (epididymitis, orchitis), secondary bacterial or fungal infection from disseminated disease, spermatocele, varicocele, or atypical testicular torsion. Our case in particular was quite severe and was likely related to the patient's immunocompromised state secondary to non-compliance with diabetes mellitus medications and diabetic ketoacidosis. Diabetes mellitus was a likely catalyst in dissemination of the pulmonary coccidioidomycosis to the genitourinary system. This case highlights the importance of considering fungal infections in the differential, especially when located in areas endemic to certain fungal infections, as swift treatment may reduce both morbidity and mortality.

\section{REFERENCES}

[1] N. M. Ampel, "Chapter 200: Coccidioidomycosis," In: D. L. Longo, A. S. Fauci, D. L. Kasper, S. L. Hauser, J. L. Jameson and J. Loscalzo, Eds., Harrison's Principles of Internal Medicine, 18th Edition, McGraw-Hill, New York, 2012. http://www.accessmedicine.com

[2] D. A. Stevens, "Coccidioidomycosis," New England Journal of Medicine, Vol. 332, No. 16, 1995, pp. 10771082. doi:10.1056/NEJM199504203321607

[3] G. J. Wise, G. S. Talluri and V. K. Marella, "Fungal Infections of the Genitourinary System: Manifestations, Diagnosis, and Treatment," Urologic Clinics of North America, Vol. 26, No. 4, 1999, pp. 701-718. doi:10.1016/S0094-0143(05)70212-3

[4] H. M. Weyrauch, F. W. Norman and J. B. Bassett, "Coccidioidomycosis of the Genital Tract," California Medicine, Vol. 72, No. 6, 1950, pp. 465-468.

[5] J. G. Rohn, J. C. Davila and T. E. Gibson, "Urogenital Aspects of Coccidioidomycosis: Review of the Literature 
and Report of Two Cases," Journal of Urology, Vol. 65, No. 4, 1951, pp. 660-667.

[6] G. Amromin and C. M. Blumenfeld, "Coccidioidomycosis of the Epididymis," California Medicine, Vol. 78, No. 2, 1953, pp. 136-138.

[7] J. M. Pace, "Coccidioidomycosis of the Epididymis," Southern Medical Journal, Vol. 48, No. 3, 1955, pp. 259260. doi:10.1097/00007611-195503000-00007

[8] H. Bodner, A. H. Howard and J. H. Kaplan, "Coccidioidomycosis of the Spermatic Cord; Roentgen TherapyReport of a Case," The Journal of the International College of Surgeons, Vol. 32, 1959, pp. 530-532.

[9] B. G. Stewart, "Epididymitis and Prostatitis due to Coccidioidomycosis: A Case Report with 5-Year Follow-Up," Journal of Urology, Vol. 91, 1964, pp. 280-281.

[10] S. Weitzner, "Coccidioidomycosis of Prostate and Epididymis - Case Report and Review of Literature," Southwest Medical, Vol. 49, No. 4, 1968, pp. 67-69.

[11] S. F. Cheng, "Bilateral Coccidioidal Epididymitis," Urology, Vol. 3, No. 3, 1974, pp. 362-363. doi:10.1016/S0090-4295(74)80124-X

[12] J. E. Gottesman, "Coccidioidomycosis of Prostate and Epididymis with Urethrocutaneous Fistula," Journal of Urology, Vol. 4, No. 3, 1974, pp. 311-314. doi:10.1016/0090-4295(74)90384-7

[13] W. T. Conner, G. W. Drach and W. C. Bucher, "Genitourinary Aspects of Disseminated Coccidioidomycosis," Journal of Urology, Vol. 113, No. 1, 1975, pp. 82-88.

[14] E. A. Petersen, B. A. Friedman, E. D. Crowder and D. Rifkind, "Coccidoidouria: Clinical Significance," Ann In- ternal Medicine, Vol. 85, No. 1, 1976, pp. 34-38. doi:10.7326/0003-4819-85-1-34

[15] K. T. Chen, "Coccidioidomycosis of the Epididymis" Journal of Urology, Vol. 130, No. 5, 1983, pp. 978-979.

[16] J. C. Liao and R. E. Reiter, "Coccidioidomycosis Presenting as Testicular Mass," Journal of Urology, Vol. 166, No. 4, 2001, p. 1396. doi:10.1016/S0022-5347(05)65785-0

[17] A. Singer, "Coccidioidomycosis of the Epididymis and Testicle," Infectious Urology, Vol. 15, No. 2, 2002, pp. 21-25.

[18] E. S. Halsey, M. S. Rasnake and D. R. Hospenthal, "Coccidioidomycosis of the Male Reproductive Tract," Mycopathologia, Vol. 159, No. 2, 2005, pp. 199-204. doi:10.1007/s11046-004-6260-0

[19] M. R. Sohail, P. E. Andrews and J. E. Blair, "Coccidioidomycosis of the Male Genital Tract," Journal of Urology, Vol. 173, No. 6, 2005, pp. 1978-1982. doi:10.1097/01.ju.0000158455.24193.12

[20] T. M. Dykes, A. B. Stone and E. D. Canby-Hagino, "Coccidioidomycosis of the Epididymis and Testis," American Journal of Roentgenology, Vol. 184, No. 2, 2005, pp. 552-553. doi:10.2214/ajr.184.2.01840552

[21] D. Ruggles, "Testicular Coccidioidomycosis," Urologic Nursing, Vol. 28, No. 2, 2008, pp. 113-114.

[22] T. M. Chiller, J. N. Galgiani and D. A. Stevens, "Coccidioidomycosis," Infectious Disease Clinics of North America, Vol. 17, No. 1, 2003, pp. 41-57. doi:10.1016/S0891-5520(02)00040-5 